\title{
Analysis of the interband optical transitions: Characterization of synthetic DNA band structure
}

\author{
Elena Díaz ${ }^{\mathrm{a}}$ \\ GISC, Departamento de Física de Materiales, Universidad Complutense, E-28040 Madrid, Spain
}

(Received 9 November 2007; accepted 29 February 2008; published online 1 May 2008)

\begin{abstract}
We analyze the band structure and interband optical transitions in a dangling backbone ladder DNA model. Using this model, semiconducting synthetic poly $(\mathrm{G})$-poly $(\mathrm{C})$ DNA is studied by means of a tight-binding model traditionally used for transport studies. Numerical calculations for optical absorption spectra are also presented. By studying the eigenstates' symmetries in uniform and nonuniform DNA chains, we conclude that, in both cases, the transitions are almost vertical in $K$ space. The optical gap turns out larger than the electronic one, and an indirect band gap electronic structure for this DNA model is revealed. The effects of the environment, which are relevant for the wet form of DNA, are taken into account by introducing disorder in the backbone levels. We demonstrate that they affect more the spectra in the case of parallel polarization of the incoming light (with respect to the molecule axis). In such a case, the closure of the gap appears for a large enough disorder. We also consider the natural helix DNA conformation and find unusual selection rules for interband optical transitions. We propose that a comparison between the obtained spectra and the experiments can provide an insight into the electronic band structure of DNA.
\end{abstract}

(C) 2008 American Institute of Physics. [DOI: 10.1063/1.2901046]

\section{INTRODUCTION}

The electronic transport in DNA molecules has attracted much attention from several scientific fields in the last years. Biological interests are mostly based on the possibility of revealing and controlling damages in DNA by means of electrical currents (see Refs. 1 and 2, and references therein for an overview). On the other hand, the physical motivation mainly originates from the search of new materials for nanotechnological aims such as wires, transistors, diodes, and all sorts of molecular electronics. ${ }^{3-6}$ Furthermore, DNA, which spontaneously assembles, ${ }^{7}$ has also been revealed as a very useful building material to organize other higher conductive nanomaterials. ${ }^{8}$

Due to such interest, several experiments on electrical transport through DNA molecules has been performed but no consistent conclusion has arisen from them yet. Indeed, depending on the sequence of nucleotide bases or on the environment conditions, such as contact, thermal, or solution effects, DNA has been revealed as a proximityinduced superconductor, ${ }^{9}$ metal, ${ }^{10-13}$ semiconductor, ${ }^{14-18}$ and insulator. $^{19,20}$

In the case of dry synthetic DNA, such as the poly $(\mathrm{G})$-poly $(\mathrm{C})$, theoretical calculations already predicted its semiconducting behavior in the $1960 \mathrm{~s}^{21}$ However, because of the difficulties in manipulating single DNA molecules, it was not definitely established by experiments until much later. ${ }^{14}$ Since this crucial experimental goal, several theoretical model $\mathrm{s}^{22-34}$ have provided a reasonable description of the semiconducting gap observed in the experiments. Although most of these models are based on the effective

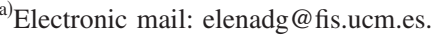

Hamiltonian models within the tight-binding approximation, there is no complete agreement about the model parameters yet.

This paper continues a previous study ${ }^{24}$ of interband optical transitions between the highest occupied molecular orbital (HOMO) and lowest unoccupied molecular orbital (LUMO) bands. This optical study can provide an insight into the electronic band structure of DNA, giving some useful information to define more realistic theoretical models. Furthermore, it allows one to characterize poly $(\mathrm{G})-\operatorname{poly}(\mathrm{C})$ DNA as a direct or indirect band gap semiconductor, which is interesting for developing new applications of DNA for optical devices as well.

To this end, we present a theoretical analysis on the selection rules for interband transitions in a dangling backbone ladder (DBL) DNA model by considering a traditional set of parameters used for transport calculations. This model was introduced by Klotsa $e t$ al. $^{22}$ as a generalization of the fishbone DNA model ${ }^{23}$ which reproduces the experimental results. ${ }^{14}$ We also present numerical results for the optical absorption spectra. The crucial effects of the environment on the DNA properties, not only in vivo but mainly in the experimental situations, are also taken into account by introducing disorder in the most external part of the molecule, the backbone molecules. The natural helix conformation of this biomolecule is also considered since it is relevant when dealing with the optical dipole moments of electronic transitions. ${ }^{24}$

The paper is organized as follows. In Sec. II, we introduce the tight-binding ladder model used to describe poly(G)-poly(C) DNA molecules. Section III discusses electronic states and band structure for this model and reveals well-defined wave function symmetries for the case of uni- 
form DBL model. These symmetries are relaxed in the nonuniform DBL model, as demonstrated in Sec. IV, by means of absorption spectra. However, in both cases, these symmetries give rise to almost vertical interband optical transitions, as presented in the same section. The effects of disorder in backbone (which is relevant for wet DNA) are analyzed in Sec. V. The impact of the helix geometry of the DNA molecule on interband optical transitions is studied in Sec. VI. These results obtained for the DBL model are compared in Sec. VI to the one previously published for a different ladder DNA model. ${ }^{24}$ We study the relationship between some features of the absorption spectra and the model parameters. Finally, in Sec. VIII, we summarize the results and their relevance to the experimental measurements necessary to infer the main characteristics of the interband optical transitions.

\section{MODEL}

We consider the DBL model introduced by Klotsa et $a .^{22}$ This model considers both base and sugar-phosphate backbone molecules. Similar models can be found in the literature. ${ }^{23-27}$ In this case, we use it to study the synthetic poly $(\mathrm{G})$-poly $(\mathrm{C})$ DNA, namely, a periodic stack of G-C base pairs. In order to study collective electronic properties of the whole DNA molecule, we restrict ourselves to one orbital per molecule and, therefore, intramolecule transitions are excluded in this study. The tight-binding Hamiltonian, which considers interstrand and intrastrand hoppings within the nearest-neighbor, approximation, is

$$
\begin{aligned}
\mathcal{H}= & \sum_{n=1}^{N}\left[\sum_{s=\mathrm{G}, \mathrm{C}}\left(\varepsilon_{n}^{s} a_{s, n}^{\dagger} a_{s, n}+\gamma_{n}^{s} b_{s, n}^{\dagger} b_{s, n}+t_{q} a_{s, n}^{\dagger} b_{s, n}\right)\right. \\
& +t_{\perp} a_{\mathrm{G}, n}^{\dagger} a_{\mathrm{C}, n}+t_{\mathrm{GG}}\left(a_{\mathrm{G}, n}^{\dagger} a_{\mathrm{G}, n+1}+a_{\mathrm{G}, n+1}^{\dagger} a_{\mathrm{G}, n}\right) \\
& \left.+t_{\mathrm{CC}}\left(a_{\mathrm{C}, n}^{\dagger} a_{\mathrm{C}, n+1}+a_{\mathrm{C}, n+1}^{\dagger} a_{\mathrm{C}, n}\right)\right] .
\end{aligned}
$$

Here, $a_{s, n}^{\dagger}\left(a_{s, n}\right), b_{s, n}^{\dagger}\left(b_{s, n}\right), \varepsilon_{n}^{s}$, and $\gamma_{n}^{s}$ denote the creation (annihilation) operators and site energies for base and backbone sites, respectively, and $s=\mathrm{G}, \mathrm{C}$.

We assume that the value of intrastrand $\left(t_{\|}\right)$hoppings is the same for both strands since they take into account the interactions between two identical basis, in both cases, $t_{\mathrm{GG}}=t_{\mathrm{CC}}=t_{\|}$. Due to the fact that poly $(\mathrm{G})$-poly $(\mathrm{C})$ is a sequence of identical base pairs, only one value for the interstrand $\left(t_{\perp}\right)$ hopping is also considered. Following Ref. 22, we neglect the hopping along the sugar-phosphate backbone but keep the coupling of the backbone to the stack of base pairs, describing it by the hopping $t_{q}$. Figure 1 shows a schematic view of a fragment of the poly $(\mathrm{G})$-poly $(\mathrm{C})$ DNA within the DBL model.

Once the model has been established, we can write down the equation for the electronic amplitudes at the bases. Let us denote these amplitudes as $\psi_{n}^{s}$, where $n=1,2, \ldots, N$ labels a base pair and the superscript $s=\mathrm{G}, \mathrm{C}$ labels the $\mathrm{G}$ and $\mathrm{C}$ strands, respectively. The total number of sites in the tightbinding model is $4 N$. From Fig. 1, it is clear that the amplitudes at backbone sites can be obtained as

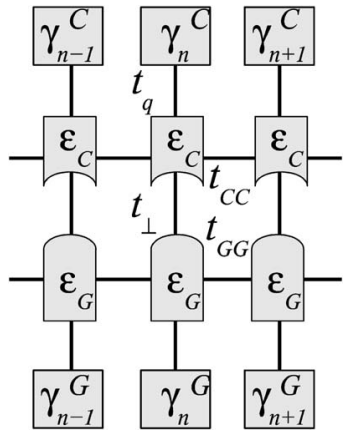

FIG. 1. Schematic view of a fragment of poly $(\mathrm{G})$-poly $(\mathrm{C})$ DNA molecules, including the sugar-phosphate backbone, according to the DBL model. Only two different hoppings are considered: The interstrand $\left(t_{\perp}\right)$ and the intrastrand $\left(t_{\mathrm{GG}}=t_{\mathrm{CC}}\right)$ ones. In addition, coupling between the backbone and the pair of bases is mediated through the hopping $t_{q}$.

$$
\phi_{n}^{s}=\frac{t_{q}}{E-\gamma_{n}^{s}} \psi_{n}^{s},
$$

where $\phi_{n}^{s}$ and $\psi_{n}^{s}$ turn out to be proportional to each other and, therefore, one can deal only with the latter. Using Eq. (2), the equations for the amplitudes at the bases are exactly given by

$$
\begin{aligned}
& \left(E-\alpha_{n}^{\mathrm{C}}\right) \psi_{n}^{\mathrm{C}}=t_{\|}\left(\psi_{n+1}^{\mathrm{C}}+\psi_{n-1}^{\mathrm{C}}\right)+t_{\perp} \psi_{n}^{\mathrm{G}}, \\
& \left(E-\alpha_{n}^{\mathrm{G}}\right) \psi_{n}^{\mathrm{G}}=t_{\|}\left(\psi_{n+1}^{\mathrm{G}}+\psi_{n-1}^{\mathrm{G}}\right)+t_{\perp} \psi_{n}^{\mathrm{C}},
\end{aligned}
$$

with renormalized site energies ${ }^{28}$

$$
\alpha_{n}^{s}=\varepsilon_{n}^{s}+\frac{t_{q}^{2}}{E-\gamma_{n}^{s}} .
$$

Here, $\varepsilon_{n}^{s}$ takes one of the two values of the base energies for the synthetic poly $(G)-\operatorname{poly}(\mathrm{C})$ DNA. The DBL model is therefore similar to a two-leg ladder model, in which the site energies of the bases [Eq. (3c)] are renormalized due to the coupling to the backbone. Such renormalization can be important when effects related to the environment-induced disorder are considered. ${ }^{22}$

\section{ELECTRONIC WAVE FUNCTIONS}

As a first approximation, we consider now a uniform DBL model, in which all base energies take the same value. Although this model cannot describe any real DNA, it is very enlightening and allows for a better understanding of the main features of the interband optical transitions qualitatively. To this end, in this section, we set $\gamma_{n}^{s}=\varepsilon_{n}^{s}=0$. The discussion of the DBL model with different site energies on each strand is presented in Sec. IV below.

Introducing the symmetric and antisymmetric combinations of amplitudes $\psi_{n}^{ \pm}=\psi_{n}^{\mathrm{C}} \pm \psi_{n}^{\mathrm{G}}$, we can decouple Eqs. (3a)-(3c) (Ref. 35) to obtain

$$
\left(E \mp t_{\perp}-\frac{t_{q}^{2}}{E}\right) \psi_{n}^{ \pm}=t_{\|}\left(\psi_{n+1}^{ \pm}+\psi_{n-1}^{ \pm}\right) \text {. }
$$

The eigenfunctions of Eq. (4) with rigid boundary conditions are easily found since they correspond to two onedimensional decoupled lattices. By inserting these solutions in Eqs. (3a)-(3c), we get the amplitudes at the bases. We 


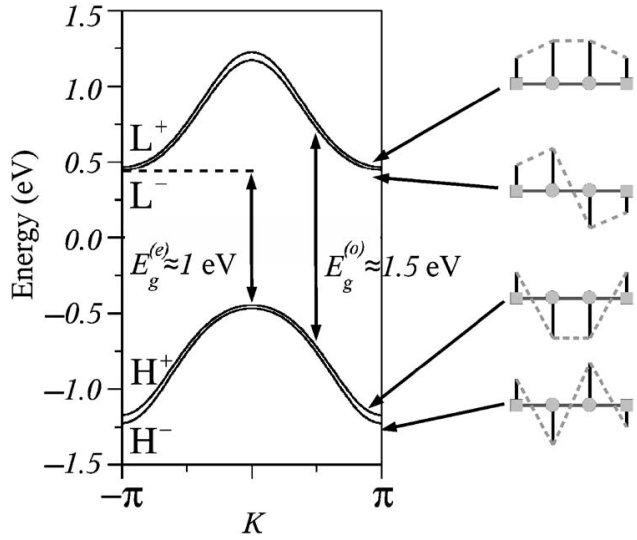

FIG. 2. HOMO $\left(\mathrm{H}^{ \pm}\right)$and LUMO $\left(\mathrm{L}^{ \pm}\right)$bands within the uniform DBL model. The right plot schematically shows the symmetries of the wave functions in the perpendicular direction. The squares and circles represent the backbone and base sites, respectively. Electronic and optical gaps are indicated by vertical arrows.

notice that solutions of Eqs. (3a)-(3c) have well-defined symmetries not only along the base-stacking direction (hereafter referred to as parallel) but also in the transversal direction (hereafter referred to as perpendicular). ${ }^{36}$ Thus, we obtain four bands of electronic states of different symmetries.

Symmetric states. The un-normalized amplitudes at the base sites are given by $\psi_{n}^{\mathrm{G}}=\psi_{n}^{\mathrm{C}}=\sin (K n)$ and the corresponding eigenenergies are

$$
E(K)=\frac{1}{2} E_{+}(K) \pm \frac{1}{2} \sqrt{E_{+}^{2}(K)+4 t_{q}^{2}}
$$

where $K=\pi k /(N+1)$ with $k=1,2, \ldots, N$, and $E_{+}(K)=t_{\perp}$ $+2 t_{\|} \cos (K)$ is the electron dispersion relation in the onedimensional lattice with on-site energies $t_{\perp}$ and hopping $t_{\|}$.

Antisymmetric states. In this case, the un-normalized amplitudes are expressed as $\psi_{n}^{\mathrm{G}}=-\psi_{n}^{\mathrm{C}}=\sin (K n)$ and the corresponding eigenenergies are

$$
E(K)=\frac{1}{2} E_{-}(K) \pm \frac{1}{2} \sqrt{E_{-}^{2}(K)+4 t_{q}^{2}}
$$

with $E_{-}(K)=-t_{\perp}+2 t_{\|} \cos (K)$.

Figure 2 shows the HOMO and LUMO bands in the DBL model, according to Eqs. (5a) and (5b). Here, we take $t_{\perp}=0.037 \mathrm{eV}, t_{\|}=0.37 \mathrm{eV}$, and $t_{q}=0.74 \mathrm{eV}$. The first value was suggested by Klotsa et al. in Ref. 22 as a SPARTAN result taking into account the weak overlap across the hydrogen bonds between the basis of a Watson-Crick pair. The rest of the hoppings were proposed by Cuniberti et al. in Ref. 23 to reproduce experimental $I-V$ curves. ${ }^{14}$ Furthermore, they are within the range of values obtained by chemical quantum calculations. $^{37,38}$

The electronic amplitudes at the backbone and base sites are also indicated. Note that Eq. (2) reduces to $\phi_{n}^{s}$ $=\left(t_{q} / E\right) \psi_{n}^{s}$ in the case of the uniform DNA. Therefore, the amplitudes $\psi_{n}^{s}$ and $\phi_{n}^{s}$ have the same sign for the LUMO band states because $E>0$, and opposite signs for the HOMO band states, since $E<0$.

\section{ABSORPTION COEFFICIENT DUE TO INTERBAND OPTICAL TRANSITIONS}

The solutions presented in the previous section will be the starting point to calculate the optical absorption coefficient due to interband transitions in DNA molecules. ${ }^{24}$ To simplify the calculations, we will restrict ourselves to zero temperature and assume that the lower (HOMO) band is full and the upper (LUMO) band is empty. Under these assumptions, the absorption coefficient due to interband transition is given by the Fermi golden rule as

$$
A(\hbar \omega) \propto \sum_{i} \sum_{f}|\langle i|\hat{e} \cdot \mathbf{r}| f\rangle|^{2} \delta\left(E_{f}-E_{i}-\hbar \omega\right),
$$

where $i$ and $f$ run over the states of the HOMO and LUMO bands, respectively. Here, $\hat{e}$ is the unit vector along the polarization direction of the incoming electromagnetic plane wave. For the sake of clarity, we now separately discuss the two independent polarizations of the incoming field.

We then focus on the square of the optical transition matrix elements $F_{i j}=|\langle i|\hat{e} \cdot \mathbf{r}| f\rangle|^{2}$ as it determines the selection rules for the optical transitions. The $\delta$-function in Eq. (6) is replaced by a square-box function with width of $10 \mathrm{meV}$ to take into account the homogeneous broadening.

\section{A. Perpendicular polarization}

The optical transition matrix element for the perpendicular polarization reduces to

$$
F_{i f} \propto\left|\sum_{n=1}^{N}\left(\psi_{n, i}^{+} \psi_{n, f}^{-}+\psi_{n, i}^{-} \psi_{n, f}^{+}\right)\right|^{2} .
$$

In this case, the symmetry of the initial and final electron states in the perpendicular direction should be different. As a consequence, only $H^{+} \rightarrow L^{-}$and $H^{-} \rightarrow L^{+}$transitions are allowed, where the superscripts refer to symmetric $(+)$ and antisymmetric $(-)$ states. Moreover, it is a matter of simple algebra to demonstrate that

$$
F_{i f} \propto \delta\left(K_{i}-K_{f}\right)
$$

namely, only vertical transitions in $K$ space are allowed. We will dicuss this issue in more detail below.

\section{B. Parallel polarization}

A similar calculation for parallel polarization yields

$$
F_{i f} \propto\left|\sum_{n=1}^{N}\left(\psi_{n, i}^{+} \psi_{n, f}^{+}+\psi_{n, i}^{-} \psi_{n, f}^{-}\right) n\right|^{2} .
$$

The initial and final electron states should have the same symmetry in the perpendicular direction to give rise to an allowed transition. There is no simple closed expression for the optical transition matrix elements in this case, but for both possible types of symmetry of the states, it is possible to demonstrate that 


$$
\begin{aligned}
F_{i f} \propto & \left|\sum_{n=1}^{N} n \sin \left(K_{i} n\right) \sin \left(K_{f} n\right)\right|^{2} \\
\propto & \left|\sum_{n=1}^{N} n\left[\cos \left(\left(K_{i}+K_{f}\right) n\right)-\sum_{n=1}^{N} \cos \left(\left(K_{i}-K_{f}\right) n\right)\right]\right|^{2} \\
\propto & \mid \frac{1}{2}\left(\frac{1-(-1)^{k_{i}-k_{f}}}{\sin ^{2}\left(\frac{K_{i}-K_{f}}{2}\right)}-\frac{1-(-1)^{k_{i}+k_{f}}}{\sin ^{2}\left(\frac{K_{i}+K_{f}}{2}\right)}\right) \\
& -(N+1)\left[(-1)^{1+k_{i}-k_{f}}-\left.(-1)^{\left.1+k_{i}+k_{f}\right]}\right|^{2}\right.
\end{aligned}
$$

where again $K_{i, f}=\pi k_{i, f} /(N+1)$ with $k_{i, f}=1,2, \ldots, N$.

The previous expression is nonzero only if $k_{i} \pm k_{f}$ is odd. Then,

$$
F_{i f} \propto\left|\sin ^{-2}\left(\frac{K_{i}+K_{f}}{2}\right)-\sin ^{-2}\left(\frac{K_{i}-K_{f}}{2}\right)\right|^{2} .
$$

Numerically, it can be checked that by increasing $\left|K_{f}-K_{i}\right|$, the optical transition matrix elements rapidly decrease. Therefore, the allowed optical transitions are almost vertical in $K$ space.

The fact that only vertical or almost vertical transitions in $K$ space are allowed has strong impact on the optical properties of DNA. In transport measurements, e.g., in a currentvoltage experiment, the electronic gap can be revealed. The latter is given by the difference of the closest band edges independent of whether they are at the same or different points of the $K$ space. If the intrastrand hoppings are of the same sign for both strands (as in the case of the DBL model), this difference is between the $\Gamma$ point $(K=0)$ of the HOMO and the $X$ point $(K=\pi)$ of the LUMO bands or vice versa (see Fig. 2 for illustration). The optical gap can be larger in the case of indirect band structure DNA due to exact or almost exact conservation of the quasimomentum requirement (interaction with phonons is not considered here). This difference is clearly observed in Fig. 3(a), where we show the absorption spectra for parallel and perpendicular polarizations. The presented results were calculated for $N=500$, $t_{\perp}=0.037 \mathrm{eV}, t_{\|}=0.37 \mathrm{eV}$, and $t_{q}=0.74 \mathrm{eV}$. The results correspond to the uniform DBL model, in which all site energies are set to zero (see Sec. III). Note that the optical gap is about $1.45 \mathrm{eV}$ for both polarizations, while the electronic gap is about $1.0 \mathrm{eV}$.

The width of the absorption band can be exactly calculated in the case of the perpendicular polarization. The energy of the lower absorption edge is obtained as the difference between $L^{-}$and $H^{+}$bands at $K=\pi / 2$. This difference is found to be $1.45 \mathrm{eV}$, which is in agreement with Fig. 3(a). Similarly, the energy of the upper absorption band edge is obtained as the difference of $L^{+}$and $H^{-}$bands at $K=0$, which gives $1.69 \mathrm{eV}$. Thus, the width of the absorption band due to interband optical transitions within the uniform model is about $250 \mathrm{meV}$.

As mentioned above, the uniform DBL model is not realistic in the sense that all site energies are set to zero

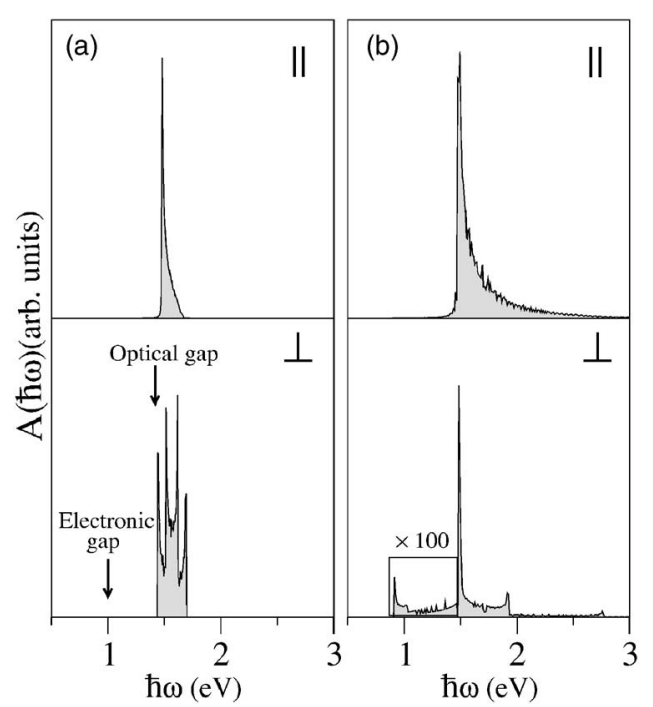

FIG. 3. Absorption spectra in (a) uniform and (b) nonuniform DBL models for parallel (upper panels) and perpendicular (lower panels) polarizations.

(see Sec. III). To ascertain whether the results obtained for the uniform DBL model are relevant to the understanding of optical transitions in real DNA, we have also considered a nonuniform DBL model by setting $\epsilon_{\mathrm{G}}=7.75 \mathrm{eV}$, $\epsilon_{\mathrm{C}}=8.87 \mathrm{eV}$, and the backbone site energy $\gamma_{\mathrm{BB}}=\left(\epsilon_{\mathrm{G}}+\epsilon_{\mathrm{C}}\right) / 2 .{ }^{22}$ Note that these energies are the base ionization potentials well established by molecular orbital calculations. ${ }^{38,39}$ On the other hand, $t_{\perp}$ and $t_{q}$ were kept the same while two different intrastrand hoppings are considered now. Thus, we keep the previous value $t_{\mathrm{GG}}=t_{\|}=0.37 \mathrm{eV}$ and we choose $t_{\mathrm{CC}}=4 t_{\mathrm{GG}}$ in order to take into account the different widths of the HOMO and LUMO bands obtained in Ref. 40. In this situation, the site energy of the two strands are different and the symmetries of the states are broken. The corresponding absorption spectra for parallel and perpendicular polarizations are shown in Fig. 3(b). As expected, the absence of symmetric and antisymmetric states relaxes the selection rules and new interband optical transitions appear. However, the new absorption band below $1.45 \mathrm{eV}$ (the optical gap in the uniform DBL) are several orders of magnitude weaker than the main band and can therefore be neglected. As a consequence, the difference between the optical and electronic gap remains in the nonuniform DBL model.

\section{EFFECTS OF DISORDER}

In this section, we consider the environmental effects on the interband optical transitions described above. The disorder can originate from interactions with a random environment of solute molecules and ions surrounding the DNA molecule. To model these interactions we vary backbone site energies in the DBL model (see Fig. 1) as $\gamma_{n}^{s}=\gamma_{\mathrm{BB}}+\Delta \gamma_{n}^{s}$, where $\gamma_{\mathrm{BB}}$ is the unperturbed value of the backbone energy, while $\Delta \gamma_{n}^{s}$ are the stochastic variables generated by a box probability distribution with zero mean value and width $w$, hereafter referred to as magnitude of disorder. We restrict ourselves to static disorder as we consider the $T=0$ case and the molecule is supposed to be embedded into a glassy host. 

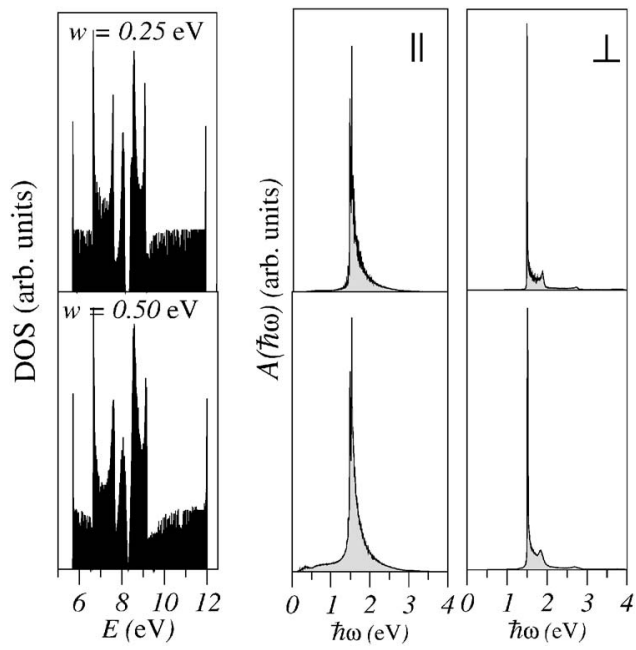

FIG. 4. Left panels show the DOS for two different magnitudes of backbone disorder indicated in each plot. The corresponding absorption spectra for parallel (middle panels) and perpendicular (right panels) polarizations are also shown. Each spectrum $A(\hbar \omega)$ comprises the average over 200 realizations of disorder for $N=200$.

Figure 4 shows the absorption spectra for two different magnitudes of the backbone disorder: $w=0.25 \mathrm{eV}$ and $w=0.50 \mathrm{eV}$ in the nonuniform DBL model. The left panel shows the corresponding density of states (DOS). At moderate disorder $(w=0.25 \mathrm{eV})$, the electronic gap is still open and the corresponding absorption spectrum broadens as compared to the case of the homogeneous DNA discussed in the previous section. Note that the absorption edge for perpendicular polarization is not sharp, reflecting the relaxation of the optical selection rules. As the magnitude of disorder increases $(w=0.5 \mathrm{eV})$ the electronic and the optical gap closes, ${ }^{24}$ as the left panel of Fig. 4 demonstrates. The effect is much more pronounced for parallel polarization.

In Fig. 5, we show quantitative estimations of the electronic and optical gaps (the latter for parallel polarization) for different magnitudes of disorder. The main difference between the two gaps occurs in the limit $w \rightarrow 0$, where the symmetries of the eigenstates involved in the allowed optical transitions are well defined. In the range $0.1 \leqslant w \leqslant 0.5 \mathrm{eV}$, the dependence of both gaps on the magnitude of disorder is

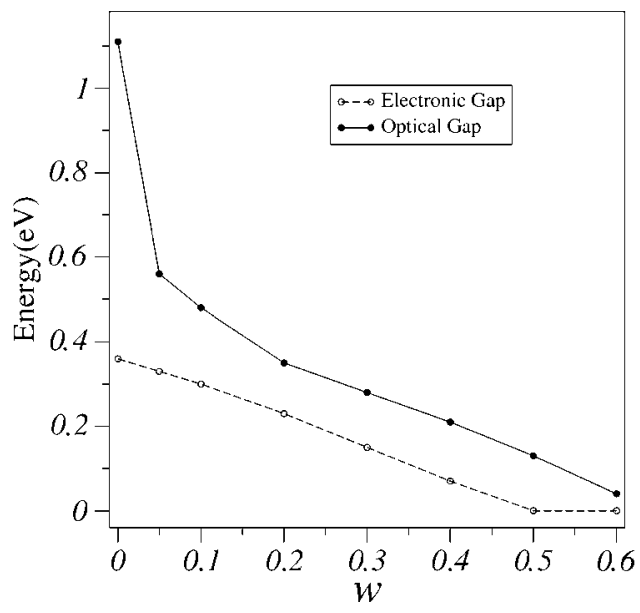

FIG. 5. Electronic and optical gaps for increasing magnitude of disorder. roughly the same and, therefore, the difference between them reaches a constant value around $0.12 \mathrm{eV}$. Indeed, when $w$ $=0.5 \mathrm{eV}$, the electronic gap is closed while the optical one is still open. Finally, if $w>0.6 \mathrm{eV}$, both gaps are completely closed.

\section{IMPACT OF THE HELIX CONFORMATION OF THE DNA STRANDS}

Up until now, we are neglecting the double helix geometry of the DNA molecule within the DBL model. However, the spatial arrangement of bases has recently been demonstrated to be crucial for transport properties in the presence of the perpendicular electric field. ${ }^{34}$ In addition, it can also be relevant when dealing with optical dipole moments of the electronic transitions. To improve the analysis, we follow Ref. 24 and take the twisted DBL model as a set of four helices. The inner layer is composed by the traditional double helix structure of DNA bases and the outer one corresponding to the backbone sites arranged in the same geometry. The coordinates of the bases along both strands can be set then as

$$
\begin{aligned}
& x_{n}^{s}=R \cos \left(\frac{2 \pi}{T} n+\beta_{s} \phi_{0}\right) \\
& y_{n}^{s}=R \sin \left(\frac{2 \pi}{T} n+\beta_{s} \phi_{0}\right) \\
& z_{n}^{s}=v n,
\end{aligned}
$$

where $n=1, \ldots, N$ and $s=\mathrm{C}, \mathrm{G}$. Here, $\beta_{\mathrm{C}}=+1$ and $\beta_{\mathrm{G}}=-1$. We consider the $\mathrm{B}$ form of the DNA with the following parameters of the double helix: Full twist period of ten base pairs, i.e., $T=10$. Finally, we set $\phi_{0}=\pi / 3$, so that the relative phase difference between the helices is $2 \phi_{0}=2 \pi / 3$, and the minor groove is one-half of the major one. The backbone site coordinates are set in the same way used for the bases but assuming a larger radius, $3 R$.

The helical conformation of the DNA strands results in unusual selection rules for interband optical transitions in the case of perpendicular polarization. ${ }^{24}$ Assume for simplicity the case of the uniform DBL model. In this simple case, the wave functions have well-defined symmetries not only along the base-stacking direction but also in the transversal direction. These symmetries give rise to selection rules for the electron momentum. It is then a matter of simple algebra to demonstrate that ${ }^{24}$

$$
F_{i f} \propto \frac{\sin ^{2}\left(\left|K_{i} \pm K_{f}\right|-2 \pi / T\right)}{\left(\left|K_{i} \pm K_{f}\right|-2 \pi / T\right)^{2}} \sim \pi \delta\left(\left|K_{i} \pm K_{f}\right|-2 \pi / T\right) .
$$

Thus, the harmonic modulation of dipole moments brings in the additional effective momentum $2 \pi / T$, which changes the selection rule from that conserving the true total momentum [see Eq. (7b)] to the conservation of the sum of the total and the effective momenta. As the result the strongest transitions appear to be indirect in $K$ space. $^{24}$ In fact, the absorption band becomes broader because of the energy structure being indirect. The amount of broadening depends on the model 


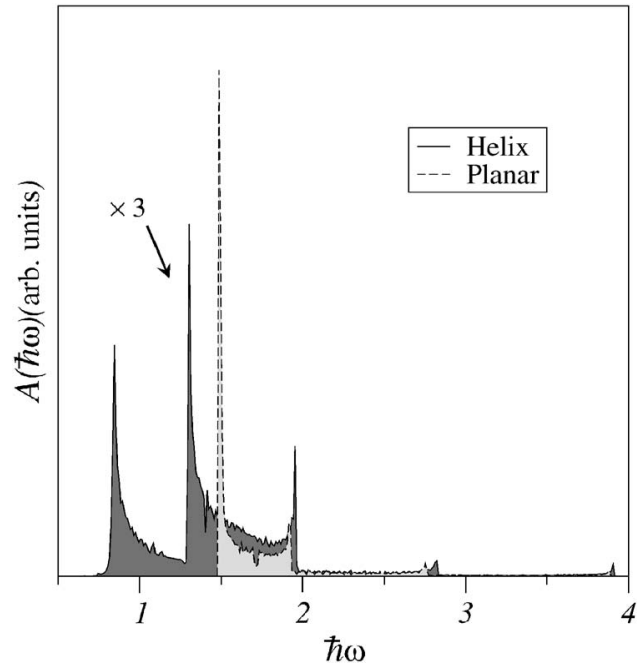

FIG. 6. Absorption spectra within the nonuniform DBL model for perpendicular polarization within the planar ladder (dashed line) and the twisted ladder (solid line) models. The number of bases at each strand is $N=500$ and the bin is $10 \mathrm{meV}$.

parameters: The smaller are the effective masses at the bottoms (tops) of the bands the larger is the effect.

Figure 6 shows the results for the nonuniform twisted DBL, with the same set of parameters used in Fig. 3. The main difference occurs for perpendicular polarization, while the absorption spectrum for parallel polarization remains unchanged. The height of the absorption spectrum is reduced as compared to the planar case. The reduction is due to the fact that dipole moments are rotating around the molecule axis as one moves along this axis, so roughly half of them are perpendicular to the field and therefore do not interact with it. Because the energy structure has an indirect band gap the helical spectrum is wider than the planar one. The line broadening is quite considerable due to relatively light masses at the tops and bottoms of the bands (large value of the longitudinal hoppings). The broadening results in the optical gap that is reduced by about $0.75 \mathrm{eV}$ as compared to the planar case.

\section{COMPARISON WITH PREVIOUS RESULTS}

In a previous work, ${ }^{24}$ an analogous study of a different ladder DNA model, referred there as simple ladder (SL) model, was performed. The model in that case consists on a simple ladder model, neglecting backbone effects. In the literature, it can be found several similar DNA models as well. $^{29-34}$

Those tight-binding model parameters are chosen to fit $a b$ initio band structure calculations for $\operatorname{poly}(\mathrm{G})-\operatorname{poly}(\mathrm{C})$ molecules. ${ }^{41}$ The intrastrands hoppings for the two strands turn out to be of opposite signs and it leads to a direct band gap electronic structure. Such a difference in the electronic structure of the DBL and SL models, namely, between a direct or an indirect band gap semiconductor energy structure can be revealed by interband optical transitions measurements.

A comparison between interband optical transitions in the case of perpendicular polarization, in both twisted mod-

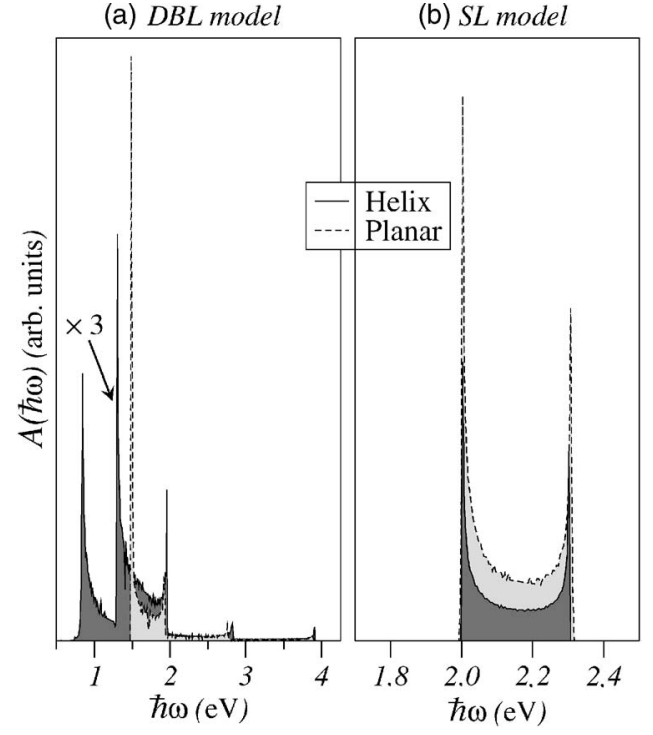

FIG. 7. Absorption spectra for perpendicular polarizations within the planar ladder (dashed line) and the twisted ladder (solid line) in (a) DBL and (b) SL models.

els, is also in order. Although the strongest transitions appear to be indirect in $K$ space, in both cases, changes of the absorption band appear as a result of the dependence of selection rules on the energy dispersion of the bands. Because of the energy structure being direct for the SL model, the helical absorption band narrows with respect to the planar case. This is just the opposite effect that we find for indirect energy structure in a DBL model (see Fig. 7). However, the amount of shrinking depends in the same way on the model parameters, giving rise to a small narrowing since effective masses are large (small dispersion) for the SL model.

\section{CONCLUSIONS}

We have studied the electronic band structure of semiconducting synthetic DNA, such as the poly $(\mathrm{G})$-poly $(\mathrm{C})$ DNA by means of a DBL DNA model traditionally used for transport calculations. ${ }^{22,23}$ It is worth mentioning that this model may be used to describe any sort of DNA molecule, natural or synthetic, using the proper parameters set for each of them, namely, site energies and hoppings between molecules. However, optical transitions can be studied only in semiconducting DNA. Therefore, we considered poly $(\mathrm{G})$ poly $(\mathrm{C})$ DNA since not only transport experiments but also density function theory (DFT) calculations, have clearly probed its semiconducting behavior. Thus, restricting ourselves to zero temperature this structure is composed by full valence band (HOMO) and a empty conduction band (LUMO) separated by a semiconducting gap.

We found well-defined symmetries for the electronic states in uniform and nonuniform DBL models. ${ }^{36}$ They lead to such selection rules for interband transitions that the optical gap turns out larger than the electronic one. We demonstrated then an indirect band gap electronic structure for this DNA model. We put forward a simple relationship of the optical gap to tight-binding model parameters and argue that 
combined optical and electric measurements can provide an insight into the underlying electronic band structure of the DNA.

Furthermore, environmental effects on the DNA molecules can be included by introducing disorder in the site energies of the backbone in the DBL model. Thus, this model can be used in many different experimental situations but mostly in natural DNA which is always in liquid solution. Indeed, there are two issues which could be modified to reproduce natural conditions, namely, the distribution and the magnitude of disorder. In this work, we have considered the effect of static disorder in backbone levels to address optical properties of wet DNA, and in this case the resulting absorption spectra have been shown as well. Disorder reduces both optical and electronic gaps. The closure of the electronic gap by the disorder provides, in particular, a simple explanation of the observed Ohmic behavior of DNA conductivity in disordered aqueous environment. ${ }^{13}$

The helical conformation of the DNA strands results in unusual selection rules for interband optical transitions in the case of perpendicular polarization of incoming light. ${ }^{24}$ Such transitions appear to be indirect in $K$ space. On these grounds, we demonstrate that the analysis of absorption spectra for parallel and perpendicular polarizations can provide valuable information on details of the DNA energy structure.

We have compared these results for the DBL model with others published elsewhere ${ }^{24}$ for a SL model. We have demonstrated that these two models, which reproduced transport experiments ${ }^{14}$ (DBL) or sophisticated ab initio calculations ${ }^{41}$ (SL) present different optical behaviors. Note the fact that considering the backbone sites within a theoretical model is not the most important point. Indeed, it can be demonstrated that a DBL model can be transformed to a SL model renormalizing the sites energies of the bases in a proper way. ${ }^{22,27,28}$ Thus, the crucial point is the tight-binding parameter set and mainly the signs of the intrastrand hoppings. In the literature, the values for these hoppings are usually positive ${ }^{37,38}$ However, they should have opposite signs ${ }^{24}$ in order to reproduce the typical curvatures of the HOMO and LUMO bands obtained by DFT calculations. ${ }^{40-42}$

Because of this inconsistency, we show, by means of the analysis of interbands optical transitions, now it could be distinguished whether the synthetic poly $(\mathrm{G})-\operatorname{poly}(\mathrm{C})$ DNA behaves as a direct or indirect semiconductor and, therefore, what is the best theoretical description for it. The difference in the amount of broadening/shrinking of these absorption spectra (see Sec. VI) may provide important information on the values of the effective masses at the top and bottom of the bands and, hence, the value of the tight-binding parameters.

Absorption spectra can be measured by means of a spectrophotometer of many DNA molecules in solution in order to intensify the absorption. Furthermore, using this experimental approach, the absorption due to the solution can be separated from that due to the DNA molecules. In this case, the measured length of the DNA chains should not exceed more than the DNA persistence length, around 200 base pairs, ${ }^{43}$ in order to deal with elongated molecules.
Although matrix elements of intramolecule excitations are several orders of magnitude larger than those involving molecular levels of different bases, ${ }^{44,45}$ the intramolecule transition energy of about $3.7 \mathrm{eV} .{ }^{45}$ observed in wet DNA is probably well above the absorption band edge for interband transitions. Thus, low-energy features in the absorption spectra due to interband transitions are relatively weak but are well separated from the strong intramolecule excitations, which suggests that they can be observed, although this could be challenging from the experimental point of view.

\section{ACKNOWLEDGMENTS}

The author thanks F. Domínguez-Adame, E. Maciá, and A. Rodríguez for helpful discussions. This work was supported by MEC (Project No. MOSAICO) and BSCH-UCM (Project No. PR34/07-15916).

${ }^{1}$ D. N. Beratan, S. Priyadarshy, and S. M. Risser, Chem. Biol. 4, 3 (1997).

${ }^{2}$ E. Boon, A. Livinstong, N. Chmiel, S. David, and J. Barton, Proc. Natl. Acad. Sci. U.S.A. 100, 12543 (2003).

${ }^{3}$ V. R. P. Bajpai and L. M. Bharadwaj, EMBO Rep. 4, 442 (2003).

${ }^{4}$ C. Dekker and M. A. Ratner, Phys. World 14, 29 (2001).

${ }^{5}$ R. G. Endres, D. L. Cox, and R. P. Singh, Rev. Mod. Phys. 76, 195 (2004).

${ }^{6}$ D. Porath, G. Cuniberti, and R. Di Felice, Top. Curr. Chem. 237, 183 (2004).

${ }^{7}$ N. C. Seeman, Nature (London) 421, 427 (2003).

${ }^{8}$ H. Yan, S. H. Park, G. Finkelstein, J. H. Reif, and T. H. LaBean, Science 301, 1882 (2003), and references therein.

${ }^{9}$ A. Yu. Kasumov, M. Kociak, S. Guéron, B. Reulet, V. T. Volkov, D. V. Klinov, and H. Bouchiat, Science 291, 280 (2001).

${ }^{10}$ Y. Okahata, T. Kobayashi, K. Tanaka, and M. J. Shimomura, J. Am. Chem. Soc. 120, 6165 (1998).

${ }^{11}$ H. W. Fink and C. Schönenberger, Nature (London) 398, 407 (1999).

${ }^{12}$ A. Rakitin, P. Aich, C. Papadopoulos, Yu. Kobzar, A. S. Vedeneev, J. S. Lee, and J. M. Xu, Phys. Rev. Lett. 86, 3670 (2001).

${ }^{13}$ O. Legrand, D. Côte, and U. Bockelmann, Phys. Rev. E 73, 031925 (2006).

${ }^{14}$ D. Porath, A. Bezryadin, S. de Vries, and C. Dekker, Nature (London) 403, 635 (2000).

${ }^{15}$ K.-H. Yoo, D. H. Ha, J.-O. Lee, J. W. Park, J. Kim, J. J. Kim, H.-Y. Lee, T. Kawai, and H. Y. Choi, Phys. Rev. Lett. 87, 198102 (2001).

${ }^{16}$ J. S. Hwang, K. J. Kong, D. Ahn, G. S. Lee, D. J. Ahn, and S. W. Hwang, Appl. Phys. Lett. 81, 1134 (2002).

${ }^{17}$ B. Q. Xu, P. M. Zhang, X. L. Li, and N. J. Tao, Nano Lett. 4, 1105 (2004).

${ }^{18}$ H. Cohen, C. Nogues, R. Naaman, and D. Porath, Proc. Natl. Acad. Sci. U.S.A. 102, 11589 (2005).

${ }^{19}$ E. Braun, Y. Eichen, U. Sivan, and G. Ben-Yoseph, Nature (London) 391, 775 (1998).

${ }^{20}$ A. J. Storm, J. van Noort, S. de Vries, and C. Dekker, Appl. Phys. Lett. 79, 3881 (2001).

${ }^{21}$ P.-O. Löwdin, Rev. Mod. Phys. 35, 724 (1963).

${ }^{22}$ D. Klotsa, R. A. Römer, and M. S. Turner, Biophys. J. 89, 2187 (2005).

${ }^{23}$ G. Cuniberti, L. Craco, D. Porath, and C. Dekker, Phys. Rev. B 65, 241314 (2002).

${ }^{24}$ E. Díaz, A. V. Malyshev, and F. Domínguez-Adame, Phys. Rev. B 76, 205117 (2007).

${ }^{25}$ K. Iguchi, Int. J. Mod. Phys. B 13, 1845 (2004).

${ }^{26}$ H. Yamada, Phys. Lett. A 332, 65 (2004); Int. J. Mod. Phys. B 18, 1697 (2004). 
${ }^{27}$ A. Rodríguez, R. A. Römer, and M. S. Turner, Phys. Status Solidi B 243, 373 (2006).

${ }^{28}$ E. Maciá and S. Roche, Nanotechnology 17, 3002 (2006).

${ }^{29}$ K. Iguchi, Int. J. Mod. Phys. B 11, 2405 (1997).

${ }^{30}$ K. Iguchi, J. Phys. Soc. Jpn. 70, 593 (2001).

${ }^{31}$ S. Roche, D. Bicout, E. Maciá, and E. Kats, Phys. Rev. Lett. 91, 228101 (2003).

${ }^{32}$ V. M. Apalkov and T. Chakraborty, Phys. Rev. B 71, 033102 (2005).

${ }^{33}$ H. Mehrez and M. P. Anantram, Phys. Rev. B 71, 115405 (2005).

${ }^{34}$ A. V. Malyshev, Phys. Rev. Lett. 98, 096801 (2007).

${ }^{35}$ The author acknowledges a hint from P. Orellana on decoupling the equations.

${ }^{36}$ The author acknowledges enlightening discussions with A. V. Malyshev on the importance of symmetries for the selection rules.

${ }^{37}$ A. Voityuk, J. Jortner, M. Bixon, and N. Roesch, J. Chem. Phys. 114,
5614 (2002).

${ }^{38}$ H. Zhang, X. Li, P. Han, X. Yang Yu, and Y. Jan, J. Chem. Phys. 117, 9 (2002).

${ }^{39}$ H. Sugiyama and I. Saito, J. Am. Chem. Soc. 118, 7063 (1996).

${ }^{40}$ P. J. de Pablo, F. Moreno-Herrero, J. Colchero, J. Gómez Herrero, P. Herrero, A. M. Baró, P. Ordejón, J. M. Soler, and E. Artacho, Phys. Rev. Lett. 85, 4992 (2000).

${ }^{41}$ E. Artacho, M. Machado, D. Sánchez-Portal, P. Ordejón, and J. M. Soler, Mol. Phys. 101, 1587 (2003).

${ }^{42}$ J. Yi, Phys. Rev. B 68, 193103 (2003).

${ }^{43}$ J. B. Hays, M. E. Magar, and B. H. Zimm, Biopolymers 8, 531 (2004).

${ }^{44}$ E. Helgren, G. Grüner, A. Omerzu, D. Mihailovic, R. Podgornik, and H. Grimm, e-print arXiv:cond-mat/0111299.

${ }^{45}$ A. Hubsch, R. G. Endres, D. L. Cox, and R. R. P. Singh, Phys. Rev. Lett. 94, 178102 (2005) 